\title{
Smart Integration of Energy Production
}

\author{
Esko K. Juuso \\ Control Engineering, Faculty of Technology, University of Oulu, Finland, esko. juuso@oulu . fi
}

\begin{abstract}
Heat is primarily important in the world's total energy consumption and especially, peak loads and seasonal variations result in problems which are difficult to efficiently come up with electricity networks. Renewable energy sources bring important new possibilities, especially for the heat energy. This paper focuses on heat energy: compact parametric models are essential in the smart integration of production in the district heating and efficient collection of the solar thermal energy. Thermal masses of the buildings are used in the peak load cutting as energy storages. The physical parameters calculated from the building information facilitate the use of different types of buildings simultaneously in the calculations. Smart adaptive control solutions extend feasible operating periods in collecting solar thermal energy. A combination of multiple control actions is essential in keeping the system in control during strong fluctuations in cloudiness and energy demand. Heat storages increase the collecting power and extend the utilisation of the solar energy utilisation over daily and seasonal periods.
\end{abstract}

Keywords: energy production, sustainable energy, district heating, solar thermal power, smart adaptive systems

\section{Introduction}

Heat represents more than half of the world's total energy consumption and three-quarters of the fuels used to meet this heat demand consist of fossil fuels (Eisentraut and Brown, 2014). Peak load cutting is highly important in reducing both production costs and environmental impacts (Hietaharju and Ruusunen, 2016). Prediction of the future energy demand and modelling the thermal behaviour of the building, i.e. the indoor temperature, have been used in (Hietaharju and Ruusunen, 2016) to cut peak loads and optimise the heat consumption.

Future trends in global energy consumption and associated environmental issues are pushing for an increased use of renewable energy sources, smaller-size power plants and distributed generation. Consumers are increasingly willing to take an active role. Storage capacities are essential in optimisation, especially solar energy which has daily and seasonal variation. Solar collector fields are combined with storage tanks []. In district heating, building thermal mass can be utilised as short term heat storage (Hietaharju and Ruusunen, 2015).

Building thermal mass and its use in peak load cutting has also been discussed in (Braun, 2003; Henze et al.,
2007; Sun et al., 2013; Kensby et al., 2015; Hagentoft and Kalagidis, 2015; Ståhl, 2009). High peak load reductions and energy savings can be achieved (Sun et al., 2013) and relatively large variations in district heating energy are tolerated in maintaining desired indoor temperature. However, the results depend highly on the thermal characteristics of the buildings. (Hagentoft and Kalagidis, 2015) The storage capacity of a building can be estimated by using thermal effusivity, which is a function of thermal conductivity and heat capacity and represents the materials ability to exchange thermal energy with its surroundings. Heavy buildings have higher thermal effusivity and offer higher energy storage capacity and more stable indoor temperatures compared with light buildings. Models are typically based either on physical principles or they are data driven or a combination of both. (Zhao and Magoulès, 2012; Kramer et al., 2012; Foucquier et al., 2013). A new parametric physical modelling approach to predict and control the indoor temperature was proposed in (Hietaharju et al., 2017).

Integration of smart grid ideas has mostly focused on electricity where the integration requires fast adaption since the storage problem is not solved for real practice. The wind power is increasing fast but the production hours are limited. The solar electricity has the same problems and as whole this leads to very volatile production situations: there are periods of over production and periods when the demand is high but hardly any electricity can be produced with wind or solar power stations. This has experienced in Finland on a winter day when it was very calm. In Germany, there have been periods of negative electricity prices when the wind and solar power production has been on a very high level. This has made the production windows of the condensing power plants difficult. Hydropower together with gas and multi-fuel power plants help in balancing.

Solar power plants should be efficient in collecting any available thermal energy in a usable form at the desired temperature range. Seasonal and daily cyclic variations as well as atmospheric conditions, such as cloud cover, humidity, and air transparency, need to be taken into account to get a fast start-up and efficient operation in varying cloudy conditions. A solar collector field is a good test platform for control methodologies (Camacho et al., 1997; Juuso, 1999; Johansen and Storaa, 2002; Cirre et al., 2007; Limon et al., 2008; Roca et al., 2011; Ayala et al., 2011). The control strategies include basic feedforward and PID schemes, adaptive control, model-based predic- 
tive control, frequency domain and robust optimal control and fuzzy logic control.

Feedforward approaches based on the energy balance use the measurements of solar irradiation and inlet temperature (Camacho et al., 1992). Lumped parameter models taking into account the sun position, the field geometry, the mirror reflectivity, the solar irradiation and the inlet oil temperature have been developed for a solar collector field (Camacho et al., 1997). A feedforward controller has been combined with different feedback controllers, even PID controllers operate for this purpose (Valenzuela and Balsa, 1998). The classical internal model control (IMC) can operate efficiently in varying time delay conditions (Farkas and Vajk, 2002). Genetic algorithms have also been used for multiobjective tuning (Bonilla et al., 2012).

Linguistic equations (LE) have been used in various industrial applications (Juuso, 1999, 2004). Modelling and control activities with the LE methodology started by the first controllers implemented in 1996 (Juuso et al., 1997) and the first dynamic models developed in 1999 (Juuso et al., 2000). The LE based dynamic simulator is an essential tool in fine-tuning of these controllers (Juuso, 2005). The LE controllers use model-based adaptation and feedforward features, which are aimed for preventing overheating, and the controller presented in (Juuso and Valenzuela, 2003) already took care of the actual setpoints of the temperature. The manual adjustment of the working point limit has improved the operation considerably. Linguistic equation (LE) control includes solutions also for cloudy conditions and varying load situations (Juuso and Yebra, 2013b). Model-based predictive control (MPC) has been used for tuning the control of large setpoint changes (Juuso, 2006). The main challenge is to handle harmful situations efficiently to reach an unattended operation as a part of a smart grid.

Heat should be strongly taken into account since it is the primary way of using energy. This paper focuses on integrating heat demands and variating operating conditions. Approaches are compared at two levels: predictive modelbased approaches for optimization in the district heating and fast intelligent control solutions in solar thermal energy collection.

\section{Smart energy network}

Smart adaptive systems provide solutions to this need: a smart energy network indicates energy production, transmission and distribution network based on a two-way communication between suppliers and consumers. A real time monitoring of the network condition, i.e. energy production, consumption and distribution, is expected to allow for a more prominent position on the market of those renewable energy resources characterised by a discontinuous and irregular generation.

The expected benefit of a decentralised energy system can be described in terms of redistribution of peak loads and the flattening of the overall power demand curve.
The negative aspects of the integrated decentralised energy production can be formulated in terms of control: the decentralised energy production can be characterised by a strong seasonal variation; each plant depends on the needs and preferences of single users; some of the production units can be subjected to weather and climate conditions. Low carbon requirements have an effect on the trend of the thermal power production. Industry requires a basic high power source which is covered by the nuclear power production.

In this paper, the energy system includes district heating, solar thermal collectors and storages (Figure 1).

\subsection{District heating}

Combined heat and power (CHP) production together with district heating is a balancing solution. Peak loads and variations in heat demand are caused by the fluctuation of outdoor temperature. Cutting peak loads in district heating network is one of such measures.

In district heating systems, the heating demand may exceeds the capacity of power plants, which means that reserve power plants needs to be started. This raises production costs (and also environmental impact) for the energy producer as more expensive oil is used for fuel instead of wood, peat or coal. The peak loads are come up by scheduling energy use. At the same time, more accurate and stable indoor temperature control could be achieved by implementing the optimization routines for energy consumption.

\subsection{Solar thermal energy}

The usage of renewable energy sources is growing as a consequence of global climate effect and enacted legislation (EED). Solar thermal energy offers considerable opportunities in this connection. The aim of solar thermal power plants is to provide thermal energy for use in an industrial process electricity generation. With fast and well damped controllers, unnecessary shutdowns and start-ups can be avoided and the plant can be operated close to the design limits (Juuso et al., 1998).

\subsection{Storage}

Heat storage is needed to cut peak loads and even the heat demand out. The storage capacity of buildings together with adaptive automation solutions offer practical solution for this. Model-based optimization solutions developed for district heating can also be applied to electric heating. This reduces the need for electricity storage.

Geothermal energy is more flexible and can be used together with solar thermal energy to utilise solar energy more efficiently.

\section{Methods}

Model-based control and optimization solutions with demand predictions are efficient in the district heating. In solar thermal applications, seasonal and daily variations could be handled with theoretical models based on energy 


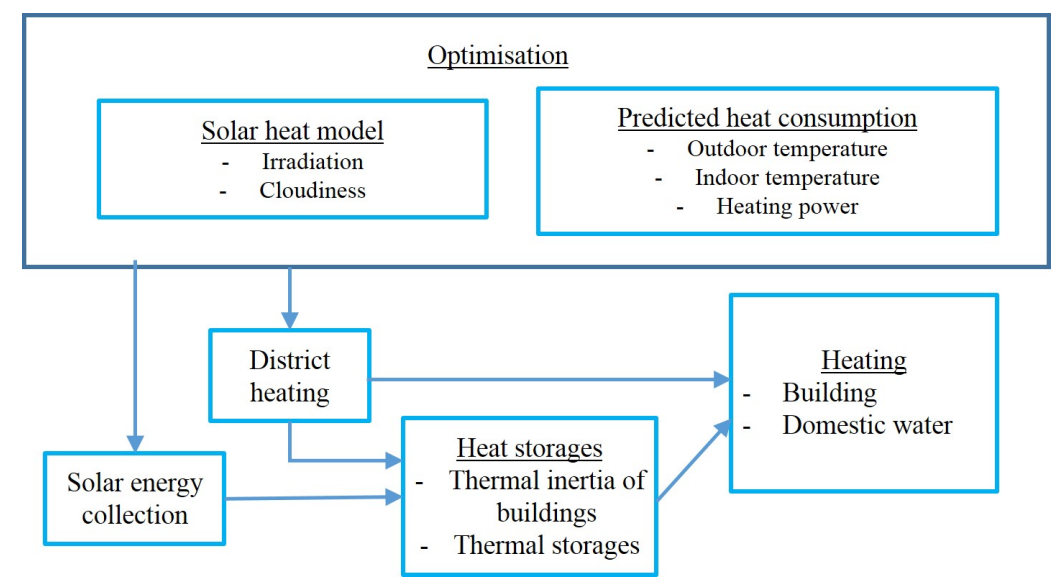

Figure 1. Optimisation of a energy system including district heating, solar thermal collectors and storages.

balances but these approaches do not provide sufficient number of operating hours. Fast variations of the operating conditions require smart adaptive control.

\subsection{Modelling}

District heating Predicting the indoor temperature evolution over time in the buildings is the key to optimizing the use of district heating. The model structure is based on Newton's cooling law: the rate of heat loss of an object is proportional to the temperature difference between the object and its surroundings. The peak load concept was introduced in (Hietaharju and Ruusunen, 2015), tested in (Hietaharju and Ruusunen, 2016) and further developed in (Hietaharju et al., 2017) to improve the efficiency of the calculations:

$$
\begin{aligned}
\Delta T_{\text {in }}(t) & =\frac{\Delta t}{C}\left[P(t-k)-U\left[T_{\text {in }}(t-1)-T_{\text {out }}(t-1)\right]\right. \\
T_{\text {in }}(t) & =T_{\text {in }}(t-1)+\Delta T_{\text {in }}
\end{aligned}
$$

where the physical parameters are obtained for buildings: $C\left(J K^{-1}\right)$ is the heat capacity and $U$ the heat loss coefficient $\left(W K^{-1}\right): U=h A$, where $h$ is the heat transfer coefficient $\left(\mathrm{Wm}^{-2} \mathrm{~K}^{-1}\right)$ and $A$ is the surface area through which the heat is being transferred $\left(m^{2}\right)$. Inputs for the model are the indoor temperature $\left(T_{i n}\right)$, outdoor temperature $\left(T_{\text {out }}\right)$ and heating power $P(W)$ which can include a lag of $k$ hours. Time step $\Delta t$ for the model is one hour. Model output is the hourly indoor temperature along the defined prediction horizon.

The physical parameters $C$ and $U$ are calculated by using ground plans and elevation drawings. Some assumptions were made about the construction materials due to insufficient information. This model was tested for two building in (Hietaharju and Ruusunen, 2016) and for a large number of buildings in (Hietaharju et al., 2017). The characteristics of these buildings are presented in (Hietaharju et al., 2017). The modelling approach can be efficiently generalised: the measured data acquired from five different types of buildings has been utilised in the model performance analysis. Use of easily available mea- surements and rough estimates for physical parameters are other important features of the model.

Solar collector field The energy balance of the collector field can be represented by expression (Valenzuela and Balsa, 1998):

$$
I_{e f f} A_{e f f}=\left(1-\eta_{p}\right) F \rho c T_{d i f f},
$$

where $I_{e f f}$ is effective irradiation $\left(\mathrm{Wm}^{-2}\right), A_{e f f}$ effective collector area $\left(\mathrm{m}^{2}\right), \eta_{p}$ a general loss factor, $F$ flow rate of the oil $\left(\mathrm{m}^{3} \mathrm{~s}^{-1}\right), \rho$ oil density $\mathrm{kgm}^{-3}, c$ specific heat of oil $\left(\mathrm{Jkg}^{-1} \mathrm{~K}^{-1}\right)$ and $T_{\text {diff }}$ temperature difference between the inlet and the outlet $\left({ }^{\circ} \mathrm{C}\right)$. The effective irradiation is the direct irradiation modified by taking into account the solar time, declination and azimuth. The density decreases and the specific heat increases resulting a nonlinear increase of the term $\rho c$ (Figure 2). In the start-up, the flow is limited by the high viscosity. The volumetric heat capacity increases very fast in the start-up stage but later remains almost constant because the normal operating temperature range is fairly narrow.
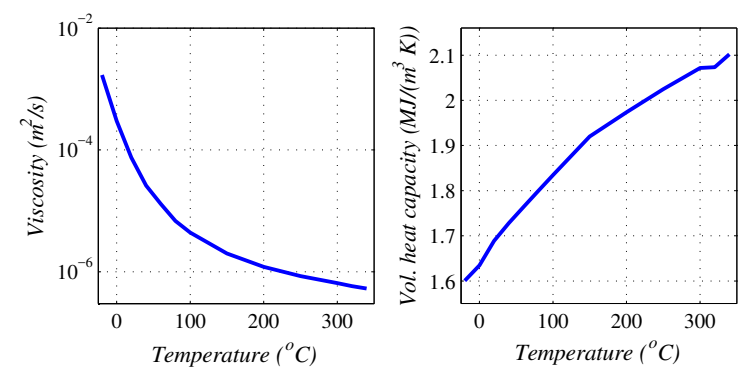

Figure 2. Oil properties (Santotherm 55) (Juuso et al., 1998).

Conventional mechanistic models do not work since there are problems with oscillations and irradiation disturbances. In dynamic LE models, the new temperature difference $\tilde{T}_{\text {diff }}(t+\Delta t)$ between the inlet and outlet depends on the irradiation, oil flow and previous temperature difference:

$$
\tilde{T}_{d i f f}(t+\Delta t)=a_{1} \tilde{T}_{d i f f}(t)+a_{2} \tilde{I}_{e f f}(t)+a_{3} \tilde{F}(t)
$$


where coefficients $a_{1}, a_{2}$ and $a_{3}$ depend on operating conditions, i.e. each submodel has different coefficients. The membership definition of the outlet temperature does not depend on time. Model coefficients and the scaling functions for $T_{\text {diff }}, I_{e f f}$ and $F$ are all model specific.

A fuzzy LE system with four operating areas is clearly the best overall model (Juuso, 2003, 2009): the simulator moves smoothly from the start-up mode via low mode to normal mode and later visits shortly in the high mode and low mode before returning to the low mode in the afternoon. Even oscillatory conditions, including irradiation disturbances, are handled correctly. The dynamic LE simulator predicts the average behaviour well but requires improvements for predicting the maximum temperature since the process changes considerably during the first hour. For handling special situations, additional fuzzy models have been developed on the basis of the FuzzyROSA method (Juuso et al., 2000).

\subsection{Smart adaptive control}

The smart control system consists of a nonlinear LE controller with predefined adaptation models, some smart features for avoiding difficult operating conditions and a cascade controller for obtaining smooth operation (Figure 3).

Data analysis The data analysis is based on the generalised norms

$$
\left\|{ }^{\tau} M_{j}^{p}\right\|_{p}=\left({ }^{\tau} M_{j}^{p}\right)^{1 / p}=\left[\frac{1}{N} \sum_{i=1}^{N}\left(x_{j}\right)_{i}^{p}\right]^{1 / p},
$$

where $p \neq 0$, is calculated from $N$ values of a sample. Several samples with length $\tau$ are used at each control step.

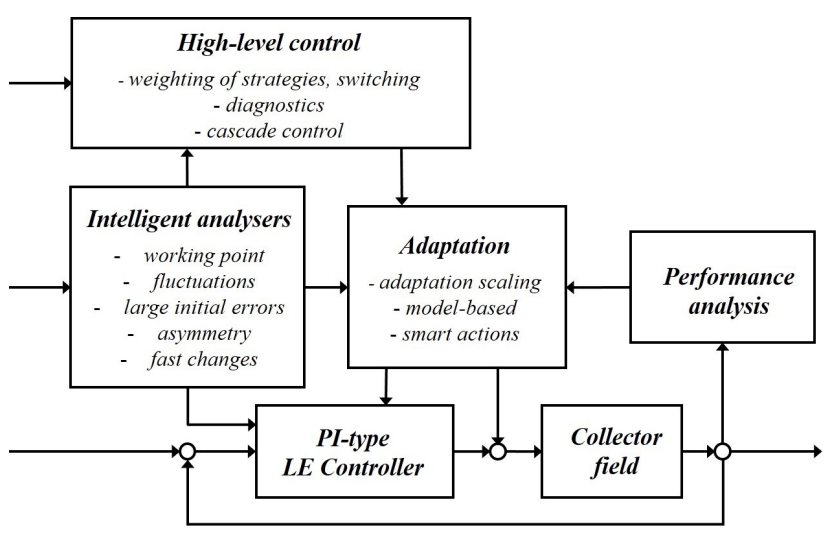

Figure 3. Smart adaptive LE control system.

Nonlinear LE control Feedback PI type controllers use errors $e_{j}(k)$ and derivatives of the errors $\Delta e_{j}(k)$ calculated for the controlled variables $j$ at each time step $k$. These real values are mapped to the linguistic range $[-2,2]$ by nonlinear scaling with variable specific membership definitions $\left(f_{e}\right)$ and $\left.f_{\Delta e}\right)$, respectively. All these functions consist of two second order polynomials and the corresponding inverse functions consist of square root functions. The scaled inputs, $\widetilde{e_{j}(k)}$ and $\widetilde{\Delta e_{j}(k)}$, are limited to the range $[-2,2]$ by using the functions only in the operating range: outside the scaled values are -2 and 2 for low and high values, respectively. The operation is enhanced with braking and asymmetry corrections.

Intelligent analysers Intelligent analyzers are used for detecting changes in operating conditions to activate adaptation and model-based control and to provide indirect measurements for the high-level control. Nonlinearities between different operating points are handled with working point models which use scaled values.

Fluctuations are detected by calculating the difference of the high and the low values of the variables as a difference of two moving generalised norms:

$$
\Delta x_{j}^{F}(k)=\left\|{ }^{K_{s} \tau} M_{j}^{p_{h}}\right\|_{p_{h}}-\left\|{ }^{K_{s} \tau} M_{j}^{p_{l}}\right\|_{p_{l}},
$$

where the orders $p_{h} \in \mathfrak{R}$ and $p_{l} \in \mathfrak{R}$ are large positive and negative, respectively. The moments are calculated from the latest $K_{s}+1$ values, and an average of several latest values of $\Delta x_{j}^{F}(k)$ is used as an indicator of fluctuations. (Juuso, 2012)

Additional indicators have been developed for high levels and fast changes to detect anomalies and avoid overshoot (Juuso and Yebra, 2014).

Adaptive control Adaptive LE control uses correction factors which are obtained from the working point value. In the solar collector field, the working point model is

$$
w p=\tilde{I}_{e f f}-\tilde{T}_{d i f f},
$$

where $\tilde{I}_{e f f}$ and $\tilde{T}_{\text {diff }}$ are obtained by the nonlinear scaling of variables: efficient irradiation $I_{e f f}$ and temperature difference between the inlet and outlet, $T_{\text {diff }}=T_{\text {out }}-T_{\text {in }}$. The outlet temperature $T_{\text {out }}$ is the maximum outlet temperature of the loops. This model handles the nonlinear effects: the volumetric heat capacity increases very fast in the start-up stage and remains almost constant in the normal operating temperatures. The predictive braking and asymmetrical actions are activated when needed. Intelligent indicators introduce additional changes of control if needed in special situations.

Model-based control Model-based control provide limits for the acceptable range of the temperature setpoint by setting a lower limit of the working point (7). The fluctuation indicators are now used for modifying the lower working point limit to react better to cloudiness and other disturbances. This overrides the manual limits if the operation conditions require that (Juuso and Yebra, 2013a, 2014). The model-based extension is an essential part in moving towards reliable operation in cloudy conditions: the control system should operate without manual interventions. The high-level control moves towards control strategies for modifying intelligent analyzers and adaptation procedures (Figure 3). 


\section{Applications}

Applications are active at two levels: predictive modelbased approaches for optimization in the district heating and fast intelligent control solutions in solar thermal energy collection.

\subsection{District heating network}

A concept for peak load cutting has been presented in (Hietaharju and Ruusunen, 2015) and tested with simulation: first for two buildings in (Hietaharju and Ruusunen, 2016) and later (Hietaharju et al., 2017) for a large number of buildings categorised in five types.

The indoor temperature models (1) and (2) have been utilised to optimise heating power in pilot buildings. First tests considered different peak load cutting scenarios based on $30 \%, 50 \%$, and $70 \%$ reduction in the heating power in the morning hours between 7 and $10 \mathrm{am}$. Load cuts were calculated from the actual measured district heating power. During the simulations, maximum allowed power was restricted accordingly during the peak load hours. Cost function for peak load cutting minimised the power consumption while keeping the indoor temperature between the control limits. This was achieved by penalizing the cost function value when the indoor temperature exceeded the limits according to the model prediction. Also the increase and decrease in the amount of heating power was restricted to prevent too large hourly power changes.

Recently, the parametric physical model has been used together with a residual model to forecast heat demand of individual buildings and the city-wide demand, aiming to provide predictive information on the heat consumption. District heating data from over 4000 different buildings at a city level has been utilised in the validation of the modelling procedure. (Hietaharju and Ruusunen, 2017)

\subsection{Solar thermal energy}

The LE control system has been tested in the solar power plant where the error variable is the deviation of the outlet temperature from the set point. The control is achieved by means of varying the flow pumped through the pipes to avoid hazardous situations, e.g. oil temperatures greater than $300{ }^{\circ} \mathrm{C}$. The goal is to reach the nominal operating temperature $180-295^{\circ} \mathrm{C}$ and keep it in changing operating conditions. The temperature increase in the field may rise up to $110^{\circ} \mathrm{C}$. (Juuso, 2011, 2012).

The intelligent indicators of the levels and fast changes of the temperatures (inlet, outlet and difference) based on intelligent indices which detect anomalies: the fast change of the inlet temperature provides feedforward information.

Fast start-up, smooth operation and efficient energy collection is achieved even in variable operating condition. The state indicators react well to the changing operating conditions and can be used in smart working point control to further improve the operation. The working point can be chosen in a way which improves the efficiency of the energy collection. A trade-off of the temperature and the flow is needed to achieve a good level for the collected power. (Juuso, 2016)

\section{Discussion}

The peak load cutting in district heating can be done efficiently with the model-based optimisation. The collection of the solar thermal energy requires smart controllers with nonlinear scaling, intelligent indicators together with adaptive and model-based extensions. Parametric systems are needed for the models and control: parameters modify the systems to different buildings and operating conditions, respectively. The models used in the district heating could be used in the solar power plant to optimise the collection demand. The smart control used in the solar application could control the heating of the buildings in varying operating conditions.

Geothermal energy is an additional energy source for district heating and individual houses. It can also enhance the use of solar thermal energy by providing efficient storages for excess solar energy. New business models based on decentralised energy production systems are arising where the end-users can simultaneously be also energy producers. This has various effects on the operation of the energy systems.

In the future, smart-grid entities formed by energy production and consumption are controlled by smart energy systems. Energy storages and coordination between electricity and heat sources are essential. A feasible production level depends on seasonal and weather condition and the dynamics of different systems need to be taken into account.

\section{Conclusions}

Parametric models are essential in the smart integration of energy production. Large networks including various types of buildings can controlled and optimised by using same compact model structures where the physical parameters are calculated from the building information. This is highly beneficial in the peak load cutting which can efficiently utilise the thermal masses of the buildings as energy storages.

Varying operating conditions are unavoidable in collecting solar thermal energy. Smart adaptive control solutions extend feasible operating periods by nonlinear scaling, intelligent analysers, predefined adaptation and model-based cascade control. The combination of multiple control actions is essential in keeping the system in control during strong fluctuations in cloudiness and energy demand. Heat storages are needed to increase the collecting power and extending the utilisation of the solar energy utilisation over daily and seasonal periods.

The efficient control and optimisation of the heat production and consumption can improve considerably the overall energy systems by reducing the peak loads and storage requirements of electricity. 


\section{Acknowledgment}

This work was supported by the Finnish Funding Agency for Innovation (TEKES) through the project KLEI (40267/13) and project ICOSLE as a part of the EU SFERA Grant Agreement 228296.

\section{References}

C. O. Ayala, L. Roca, J. L. Guzman, J. E NormeyRico, M. Berenguel, and L. Yebra. Local model predictive controller in a solar desalination plant collector field. Renewable Energy, 36:3001-3012, 2011. doi: 10.1016/j.renene.2011.03.037.

J. Bonilla, , L. J. Yebra, S. Dormido, and E. Zarza. Parabolic-trough solar thermal power plant simulation scheme, multi-objective genetic algorithm calibration and validation. Solar Energy, 86:531-540, 2012. doi: 10.1016/j.solener.2011.10.025.

J. E. Braun. Load control using building thermal mass. J. Sol. Energ.-T. Asme., 125:292-301, 2003. doi:10.1115/1.1592184.

E. Camacho, M. Berenguel, and F. R. Rubio. Adaptive Control of Solar Plants. Springer, London, 1997.

E.F Camacho, F.R. Rubio, and F.M. Hughes. Self-tuning control of a solar power plant with a distributed collector field. IEEE System Control Magazine, 12(2):72-78, 1992.

C. M. Cirre, M. Berenguel, L. Valenzuela, and E. Camacho. Feedback linearization control for a distributed solar collector field. Control engineering Practice, 15:1533-1544, 2007. doi: 10.1016/j.conengprac.2007.03.002.

A. Eisentraut and A. Brown. Heating without global warming - Market developments and policy considerations for renewable heat. IEA, Paris, 2014.

I. Farkas and I. Vajk. Internal model-based controller for a solar plant. In L. Basanez and J. A. de la Puente, editors, Proceedings of the 15th Triennial World Congress, Barcelona, Spain, July 21-26, 2011, pages 49-54. IFAC, 2002. http://www.ifacpapersonline.net/.

A. Foucquier, S. Robert, F. Suard, L. StÃl'phan, and A. Jay. State of the art in building modelling and energy performances prediction: A review. Renew. Sustain. Energy Rev., 23:272-288, 2013. doi:10.1016/j.rser.2013.03.004.

C.-E. Hagentoft and A. S. Kalagidis. Effect smart solutions for district heating networks based on energy storage in buildings. impact on indoor temperatures. Energy Procedia, 78: 2244-2249, 2015. doi:10.1016/j.egypro.2015.11.346.

G. P. Henze, T. H. Le, A. R. Florita, and C. Felsmann. Sensitivity analysis of optimal building thermal mass control. J. Sol. Energ.-T. Asme., 129:473-485, 2007. doi:10.1115/1.2770755.

P. Hietaharju and M. Ruusunen. A concept for cutting peak loads in district heating. In Automaatio XXI seminar, March 17-18, 2015, Helsinki, Finland, 2015.
P. Hietaharju and M. Ruusunen. Peak load cutting in district heating network. In 9th EUROSIM Congr. Model. Simul., Oulu, pages 140-144, 2016.

P. Hietaharju and M. Ruusunen. Heat demand forecasting by combining a parametric physical and a non-parametric model. Unpublished, 2017.

P. Hietaharju, M. Ruusunen, and K. Leiviskä. A parametric physical model for indoor temperature prediction in buildings. Submitted, 2017.

T. A. Johansen and C. Storaa. Energy-based control of a distributed solar collector field. Automatica, 38:1191-1199, 2002.

E. K. Juuso. Fuzzy control in process industry: The linguistic equation approach. In H. B. Verbruggen, H.-J. Zimmermann, and R. Babuška, editors, Fuzzy Algorithms for Control, International Series in Intelligent Technologies, volume 14 of International Series in Intelligent Technologies, pages 243300. Kluwer, Boston, 1999. doi: 10.1007/978-94-011-44056_10.

E. K. Juuso. Intelligent dynamic simulation of a solar collector field. In A. Verbraeck and V. Hlupic, editors, Simulation in Industry, 15th European Simulation Symposium ESS 2003, pages 443-449. SCS, Gruner Druck, Erlangen, Germany, 2003.

E. K. Juuso. Integration of intelligent systems in development of smart adaptive systems. International Journal of Approximate Reasoning, 35(3):307-337, 2004. doi: 10.1016/j.ijar.2003.08.008.

E. K. Juuso. Modelling and control of a solar thermal power plant. In P.Horacek, M. Simandl, and P. Zitek, editors, Proceedings of 16th Triennial World Congress, Prague, July 3-8, 2005, pages 368-373. IFAC, 2005. doi: 10.3182/200507036-CZ-1902.01790.

E. K. Juuso. Modelling and simulation in development and tuning of intelligent controllers. In I. Troch and F. Breitenecker, editors, Proceedings of 5th Vienna Symposium on Mathematical Modelling, February 8-10, 2006, Vienna, Austria, Argesim Report 30, pages 6-1 - 10. Argesin Verlag, Vienna, 2006. ISBN 3-901608-30-3.

E. K. Juuso. Dynamic simulation of solar collector fields in changing operating conditions. In B. Elmegaard, Christian Veje, Mads Pagh Nielsen, and Tommy Mølbak, editors, Proceedings of SIMS 50 - the 50th International Conference of Scandinavian Simulation Society, October 7-8, Fredericia, Denmark, pages 341-348. DTU, Lungby, Denmark, 2009.

E. K. Juuso. Recursive tuning of intelligent controllers of solar collector fields in changing operating conditions. In S. Bittani, A. Cenedese, and S. Zampieri, editors, Proceedings of the 18th World Congress The International Federation of Automatic Control, Milano (Italy) August 28 September 2, 2011, pages 12282-12288. IFAC, 2011. doi: 10.3182/20110828-6-IT-1002.03621.

E. K. Juuso. Model-based adaptation of intelligent controllers of solar collector fields. In I. Troch and F. Breitenecker, editors, 
Proceedings of 7th Vienna Symposium on Mathematical Modelling, February 14-17, 2012, Vienna, Austria, Part 1, volume 7, pages 979-984. IFAC, 2012. doi: 10.3182/201202153-AT-3016.00173.

E. K. Juuso. Intelligent control of a solar thermal power plant - adaption in varying conditions. In 2016 5th International Conference on Power Science and Engineering, Venice, Italy, 14-17 December 2016, 2016.

E. K. Juuso and L. Valenzuela. Adaptive intelligent control of a solar collector field. In Proceedings of Eunite 2003 - European Symposium on Intelligent Technologies, Hybrid Systems and their implementation on Smart Adaptive Systems, July 10-11, 2003, Oulu, Finland, pages 26-35. Wissenschaftsverlag Mainz, Aachen, 2003.

E. K. Juuso and L. J. Yebra. Model-based intelligent control of a solar energy collector field. In Proceedings - 8th EUROSIM Congress on Modelling and Simulation, EUROSIM 2013, 10-13 September, 2013, Cardiff, UK, pages 513518, 2013a. URL www. scopus. com. doi: 10.1109/EUROSIM.2013.92.

E. K. Juuso and L. J. Yebra. Optimisation of solar energy collection with smart adaptive control. In IECON Proceedings (Industrial Electronics Conference),10-14 November, 2013, Vienna, Austria, pages 7938-7943, 2013b. URL www. scopus . com. doi: 10.1109/IECON.2013.6700459.

E. K. Juuso and L. J. Yebra. Smart adaptive control of a solar collector field. In IFAC Proceedings Volumes (IFACPapersOnline), volume 19, pages 2564-2569, 2014. URL www. scopus. com.

E. K. Juuso, P. Balsa, and K. Leiviskä. Linguistic Equation Controller Applied to a Solar Collectors Field. In Proceedings of the European Control Conference -ECC'97, Brussels, July 1 - 4, 1997, volume Volume 5, TH-E I4, paper 267 (CD-ROM), page 6 pp., 1997.

E. K. Juuso, P. Balsa, and L. Valenzuela. Multilevel linguistic equation controller applied to a $1 \mathrm{MW}$ solar power plant. In Proceedings of the ACC'98, Philadelphia, PA, June 24-26, 1998, volume 6, pages 3891-3895. ACC, 1998.

E. K. Juuso, D. Schauten, T. Slawinski, and H. Kiendl. Combination of linguistic equations and the fuzzy-ROSA method in dynamic simulation of a solar collector field. In L. Yliniemi and E. Juuso, editors, Proceedings of TOOLMET 2000 Symposium - Tool Environments and Development Methods for Intelligent Systems, Oulu, April 13-14, 2000, pages 63-77, Oulu, 2000. Oulun yliopistopaino.

J. Kensby, A. Tr?schel, and J.-O. Dalenb?ck. Potential of residential buildings as thermal energy storage in district heating systems - results from a pilot test. Appl. Energ., 137:773-781, 2015. doi:10.1016/j.apenergy.2014.07.026.

R. Kramer, J. van Schijndel, and H. Schellen. Simplified thermal and hygric building models: A literature review. Front. Archit. Res., 1:318-325, 2012. doi:10.1016/j.foar.2012.09.001.

D. Limon, I. Alvarado, T. Alamo, M. Ruiz, and E. F. Camacho. Robust control of the distributed solar collector field ACUREX using MPC for tracking. In Proceedings of 17th IFAC World Congress, Seoul, Korea, July 6-11, 2008, volume 17, pages 958-963. IFAC, 2008. http://www.ifacpapersonline.net/.

L. Roca, J. L. Guzman, J. E. Normey-Rico, M. Berenguel, and L. Yebra. Filtered smith predictor with feedback linearization and constraints handling applied to a solar collector field. Solar Energy, 85:1056-1067, 2011. doi: 10.1016/j.solener.2011.02.026.

F. Ståhl. Influence of thermal mass on the heating and cooling demands of a building unit. $\mathrm{PhD}$ thesis, Chalmers University of Technology, Sweden, 2009.

Y. Sun, S. Wang, F. Xiao, and D. Gao. Peak load shifting control using different cold thermal energy storage facilities in commercial buildings: A review. Energ. Convers. Manage, 71: 101-114, 2013. doi:10.1016/j.enconman.2013.03.026.

L. Valenzuela and P. Balsa. Series and parallel feedforward control schemes to regulate the operation of a solar collector field. In Proceedings of the 2nd User Workshop Training and Mobility of Researchers Programme at Plataforma Solar de Almeria, November 10-11, 1998, pages 19-24. Ciemat, 1998.

H. Zhao and F. Magoulès. A review on the prediction of building energy consumption. Renew. Sustain. Energy Rev., 16:35863592, 2012. doi:10.1016/j.rser.2012.02.049. 